\title{
GRP78 and GAL3, differentially regulated by lymph node homogenates, as potential biomarkers for lymph node metastasis in mouse hepatocellular carcinoma cells
}

\author{
WENJUN ZHU ${ }^{1}$, LAWRENCE OWUSU ${ }^{1}$, SHIZHU ZANG $^{1}$, YUNJUAN ZHANG $^{1}$, YI XIN $^{1}$ and CHAO YAN $^{2}$ \\ ${ }^{1}$ Department of Biotechnology, Dalian Medical University, Dalian, Liaoning116044; \\ ${ }^{2}$ School of Pharmacy, Shanghai Jiao Tong University, Shanghai 200240, P.R. China
}

Received June 5, 2012; Accepted August 31, 2012

DOI: $10.3892 / \mathrm{ol} .2012 .915$

\begin{abstract}
In order to systematically evaluate the influence of lymph nodes (LNs) in lymph node metastases (LNM) of hepatocellular carcinoma (HCC), we set up a new in vitro model in which Hca-F and Hca-P cells were cultured in medium containing lymph node homogenates (LNHs). Differential protein expression was measured by two-dimensional gel electrophoresis (2-DE) combined with matrix-assisted laser desorption/ionization time-of-flight/time-of-flight mass spectrometry (MALDI TOF/TOF MS). Results from protein identification revealed two metastatic correlative proteins, 78-kDa glucose-regulated protein (GRP78) and galectin-3 (GAL3). Western blotting confirmed that GRP78, a protein positively correlated with metastasis, increased 2.4-fold in Hca-F cells but decreased to almost a half in Hca-P cells $(\mathrm{P}<0.05)$. However, GAL3, a protein negatively correlated with metastasis, was decreased by a half in Hca-F cells but slightly increased non-significantly in Hca-P cells. Thus, our results reveal that some components of LNHs may facilitate a permissive environment for cancer cells with high metastasis potential to eventually metastasize. GRP78 and GAL3 may serve as potential biomarkers for the diagnosis of LNM in HCC.
\end{abstract}

Correspondence to: Professor Yi Xin, Department of Biotechnology, Dalian Medical University, 9 West Section, Lvshun South Road, Dalian, Liaoning 116044, P.R. China

E-mail: jimxin@hotmail.com

Abbreviations: EMT, epithelial-mesenchymal transition; FBS, fetal bovine serum; GRP78, 78-kDa glucose-regulated protein; GAL3, galectin-3; HCC, hepatocellular carcinoma; LN, lymph node; LNH, lymph node homogenate; RPMI-1640, Rosewell Park Memorial Institute-1640 medium

Key words: lymph node microenvironment, hepatocellular carcinoma metastases, two-dimensional gel electrophoresis, GRP78, GAL3

\section{Introduction}

Hepatocellular carcinoma (HCC) is one of the most common types of malignant tumors and the third leading cause of cancer mortality worldwide (1). Currently, surgery is the preferred treatment method for liver cancer, but the five-year survival rate remains extremely low. Autopsy studies confirm that nearly one-third of all HCC patients have lymph node metastasis (LNM), which is the leading cause for distant metastasis and mortality; however, the molecular mechanisms of LNM from liver cancer remain unclear (2).

Hca-F and Hca-P cells are gynogenetic HCC cell lines generated from mice. They are well-characterized with different metastasis potentials exclusive to lymph nodes when inoculated subcutaneously in 615 mice. Hca-F cells have a high metastatic potential (LNM rate $>75 \%$ ), while Hca-P cells have low metastatic potential (LNM rate <25\%) $(3,4)$. Therefore, Hca-F and Hca-P cell lines were more appropriate for investigating the mechanisms of LNM in comparison to clinical samples, since high metastatic subclone cells exist in primary cancers as a minority (5).

The critical role of the cancerous microenvironment (cellular and non-cellular) is increasingly recognized as an important factor markedly influencing cancer development and metastasis $(6,7)$. The tumor microenvironment plays a decisive role in regulating the process of hepatocarcinogenesis, epithelial-mesenchymal transition (EMT), tumor invasion and metastasis (8). Additionally, global gene expression profiling of HCC has revealed that the tumor microenvironment is also an important factor in the biological and prognostic classification of HCC. The tumor microenvironment can be classified into cellular and non-cellular components. The major cellular components include fibroblasts, hepatic stellate cells, immune cells and endothelial cells. These cells produce the non-cellular components including the extracellular matrix (ECM) proteins, inflammatory cytokines, proteolytic enzymes and growth factors, which modulate the biological behavior of HCC by their effects on cancer signaling pathways in tumor cells and markedly impact tumor invasion and metastasis (8). Certain investigations of the tumor microenvironment have made significant advancements, but have mainly focused on the effect(s) of individual cellular compo- 
nents on tumor cells. However, the interactions between the tumor cells and the surrounding components have not been comprehensively and systematically demonstrated. Thus, the actual progress and mechanism of metastasis is difficult to identify.

In current studies, certain candidate genes for LNM were revealed by identifying genes with different expression levels in the Hca-F and Hca-P cell lines $(3-5,9)$. However, LNM is a dynamic process and one limitation of such studies (or the platform for such studies) is that the active changes related to LNM were not revealed. It would be necessary in the process of HCC cells encountering the lymphatic environment and growing with lymph node components. Therefore, we used an in vitro model where HCC cells with varying metastasis potential were grown with lymph node components in order to gain an insight into the possible and favorable $\mathrm{LN}$ niche condition(s) for metastasis.

\section{Materials and methods}

Cell culture and animals. Mouse HCC cell lines, Hca-F and Hca-P (established by the Department of Pathology, Dalian Medical University, Dalian, China), were grown in the abdominal cavity of 8-10-week-old inbred 615 mice (males provided by the Animal Facility of Dalian Medical University) for $\sim$ seven days (5). Cells were harvested and cultured in Rosewell Park Memorial Institute-1640 medium (RPMI-1640; Gibco BRL, Gaithersburg, MD, USA) supplemented with antibiotics (100 U/ml penicillin and $100 \mathrm{~g} / \mathrm{ml}$ streptomycin; Gibco BRL) and 10\% heat-inactivated fetal bovine serum (FBS; Gibco BRL), and incubated in a humidified incubator at $37^{\circ} \mathrm{C}$ with $5 \% \mathrm{CO}_{2}$ for one day. The study was approved by the ethics committee of Dalian Medical University, Dalian, China.

Lymph node homogenates (LNHs). The lymph nodes from inbred 615 mice were rinsed thoroughly in PBS (pH 7.4) and homogenized using a homogenizer in serum-free RPMI-1640. The homogenate was centrifuged at $2000 \mathrm{x} \mathrm{g}$ for $10 \mathrm{~min}$ at room temperature, and the supernatant was quantified using Bradford's protein assay and immediately used for subsequent experiments.

Two-dimensional gel electrophoresis (2-DE) sample preparation, running and image analysis. Hca-F cells $\left(2 \times 10^{6}\right)$ were collected, washed and cultured in $3 \mathrm{ml}$ serum-free RPMI-1640 (control) and $3 \mathrm{ml}$ serum-free RPMI-1640 with $10 \mathrm{mg} / \mathrm{ml} \mathrm{LNH}$, at $37^{\circ} \mathrm{C}$ with $5 \% \mathrm{CO}_{2}$ for $24 \mathrm{~h}$. Cells were then completely washed through serum-free RPMI1640. Cellular protein was extracted using strong radio immunoprecipitation assay (RIPA) lysis buffer containing $50 \mathrm{mM}$ TrisCl (pH 7.4), $150 \mathrm{mM} \mathrm{NaCl}, 1 \%$ Triton X-100, $1 \%$ sodium deoxycholate and $0.1 \%$ sodium dodecyl sulfate (SDS) (Beyotime Institute of Biotechnology, Haimen, Jiangsu, China) and purified with ReadyPrep ${ }^{\text {TM }}$ 2-D Cleanup Kit (Bio-Rad Laboratories, Inc., Hercules, CA, USA). The purified proteins were solubilized in 2-D rehydration buffer containing $7 \mathrm{M}$ urea, $2 \mathrm{M}$ thiourea, 4\% 3-[(3-cholamidopropyl)dimethylammonio]-1-propanesulfonic acid (CHAPS), $20 \mathrm{mM}$ dithiothreitol (DTT) and 2\% immobilized
$\mathrm{pH}$ gradient (IPG) buffer. The protein samples were then aliquoted and stored at $-80^{\circ} \mathrm{C}$ until use.

IPG gel strips (GE Healthcare, Piscataway, NJ, USA), $7 \mathrm{~cm}$ in size with a $\mathrm{pH}$ range of 3-10 (linear), were rehydrated in $125 \mu \mathrm{l}$ rehydration buffer containing $600 \mu \mathrm{g}$ of proteins at $50 \mathrm{~V}$ for $12 \mathrm{~h}$ at $20^{\circ} \mathrm{C}$. Isoelectric focusing (IEF) was conducted using a Protean IEF Cell (Bio-Rad Laboratories, Inc.). Proteins were focused at $200 \mathrm{~V}$ for $1 \mathrm{~h}, 500 \mathrm{~V}$ for $1 \mathrm{~h}$ and $800 \mathrm{~V}$ for $1 \mathrm{~h}$. A gradient of $800-8,000 \mathrm{~V}$ was then applied for $30 \mathrm{~min}$, and focusing was continued at 8,000 V for $2.5 \mathrm{~h}$.

Following IEF, IPG gel strips were equilibrated in an equilibration buffer, containing $6 \mathrm{M}$ urea, $50 \mathrm{mM}$ Tris- $\mathrm{HCl}$ (pH 8.8), $2 \%$ SDS, $30 \%$ glycerol and a trace of bromophenol blue, including 1\% DTT, for $10 \mathrm{~min}$ whilst being agitated. Before being transferred onto a $12 \%$ polyacrylamide gel, gels were transferred into an equilibration solution containing $4.5 \%$ iodoacetamide and equilibrated for $10 \mathrm{~min}$. Separation of the second dimension was carried out at a current of $5 \mathrm{~mA} / \mathrm{gel}$ for $1 \mathrm{~h}$ and $10 \mathrm{~mA} / \mathrm{gel}$ thereafter. For each sample, the 2-DE was repeated three times.

Following SDS-polyacrylamide gel electrophoresis (PAGE), gels were fixed in $30 \%$ ethanol and $10 \%$ acetic acid for $15 \mathrm{~min}$ at room temperature, rinsed three times in ultrapure water and silver stained using Pierce Silver Stain for Mass Spectrometry kit (Thermo Fisher Scientific, Inc., Fremont, CA, USA) according to the manufacturer's protocol. Images were captured using ChemiDoc XRS image documentation system and analyzed using PDQust 8.0 (Bio-Rad Laboratories, Inc.). Protein spots of interest, with an average ratio of $>2.0$ or $<-2.0(\mathrm{P}<0.05)$, were selected for protein identification by matrix-assisted laser desorption/ionization time-of-flight mass spectrometry (MALDI-TOF MS).

In-gel protein digestion and MALDI-TOF MS. Protein spots of interest were manually excised from gels and washed twice in ultra pure water in 96 -cell boards for $10 \mathrm{~min}$. The gel pieces were dehydrated completely with acetonitrile (ACN) and dried in a vacuum centrifuge for $10 \mathrm{~min}$. Subsequently, the gel pieces were incubated in $10 \mathrm{mM}$ DTT at $56^{\circ} \mathrm{C}$ for $1 \mathrm{~h}$ and in $55 \mathrm{mM}$ IAM in a dark room for $45 \mathrm{~min}$, then washed with $25 \mathrm{mM} \mathrm{NH}_{4} \mathrm{HCO}_{3}$ for $2 \times 10 \mathrm{~min}, 25 \mathrm{mM} \mathrm{NH}_{4} \mathrm{HCO}_{3} / 50 \% \mathrm{ACN}$ for $2 \times 10 \mathrm{~min}$ and $100 \% \mathrm{ACN}$ for $10 \mathrm{~min}$. Gels were then dehydrated completely with ACN and dried in a vacuum centrifuge for $10 \mathrm{~min}$. Proteins in gel pieces were rehydrated in $10 \mathrm{ng} / \mathrm{ml}$ trypsin (Roche Diagnostics GnbH, Mannheim, Germany) in $25 \mathrm{mM} \mathrm{NH}_{4} \mathrm{HCO}_{3}$ and incubated overnight at $37^{\circ} \mathrm{C}$. Digestion was terminated using $0.1 \%$ TFA. Once mixed and shocked, the mixtures were centrifuged. Supernatants ( $3 \mu \mathrm{l})$ were spotted onto the sample plates twice and dried in air, followed by a spotting matrix (70\% ACN/0.1\% TFA with $4 \mathrm{mg} / \mathrm{ml}$ a-cyano-4-hydroxycinnamic acid). Desalination was conducted twice by spotting $1 \mu 10.1 \%$ TFA on the sample plate and aspirating. Peptide masses were determined using an Ultraflex TOF/TOF MS (Bruker Daltonics, Inc., Billerica, MA, USA). The N2 laser (337 nm) was run at $100 \mu \mathrm{J}$ and $1 \mathrm{nsec}$ pulse width. Full scan MS were collected from 700-3500 m/z.

Database searching. The results of MALDI-TOF MS were analyzed by the 'Calibrate Peptide Stanard-zk. FAMS 


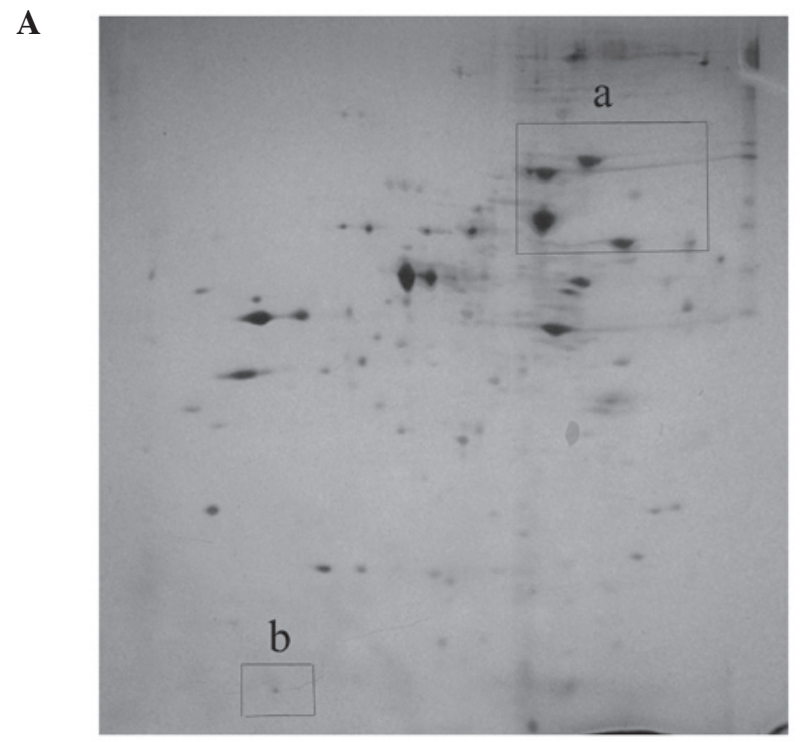

B

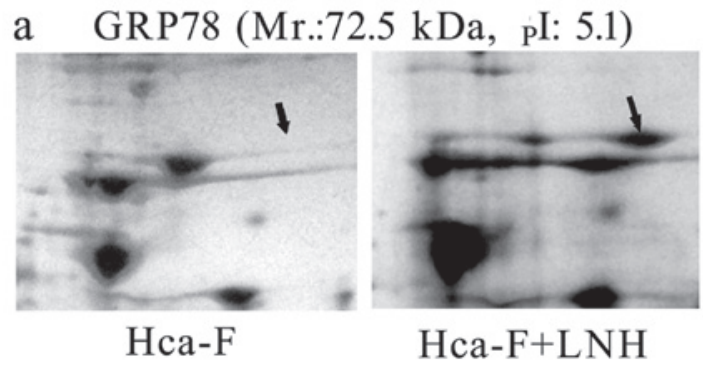

b GAL3 (Mr.: $\left.14.3 \mathrm{kDa},{ }_{\mathrm{P}} \mathrm{I}: 9.8\right)$

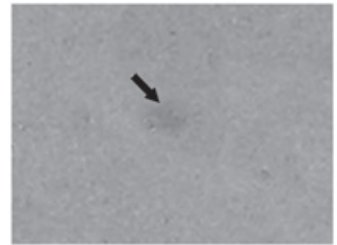

Hca-F

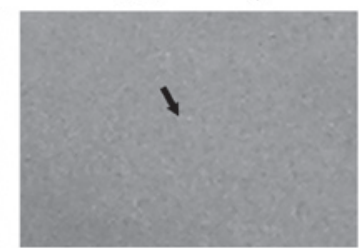

Hca-F+LNH
Figure 1. (A) Representative 2-DE proteome map of Hca-F cells. (B) Regulated protein expression of (a) GRP78 and (b) GAL3, from (A), in Hca-F cells incubated with or without $\mathrm{LNH}$ for $24 \mathrm{~h}$. Arrows indicate protein spots of interest in the regions (a) and (b). GRP78, 78-kDa glucose-regulated protein precursor; $\mathrm{Mr}$, molecular mass; $\mathrm{pI}$, isoelectric point; $\mathrm{LNH}$, lymph node homogenates; GAL3, galectin-3; 2-DE, two-dimensional gel electrophoresis.

Method' using flexanalysis software (Bruker Daltonics, Inc.). Peptide mass lists were searched against online databases in the National Center for Biotechnology Information (NCBI) using the MASCOT database search engine 2.1 (Matrix Science, London, UK). A MASCOT score $>82$ was considered to indicate a statistically significant difference $(\mathrm{P}<0.05)$.

Western blot analysis. Cellular proteins were separately extracted from $2 \times 10^{7} \mathrm{Hca}-\mathrm{F}$ and Hca-P cells using RIPA lysis buffer (Beyotime Institute of Biotechnology). The extracted proteins (40 $\mu \mathrm{g}$ of total protein) were subjected to $12 \%$ SDS-PAGE and blotted onto nitrocellulose membranes (Invitrogen Life Technologies, Carlsbad, CA, USA). Following incubation in $1 \%$ bovine serum albumin (BSA) for $1 \mathrm{~h}$, the blotted membranes were incubated with primary antibodies
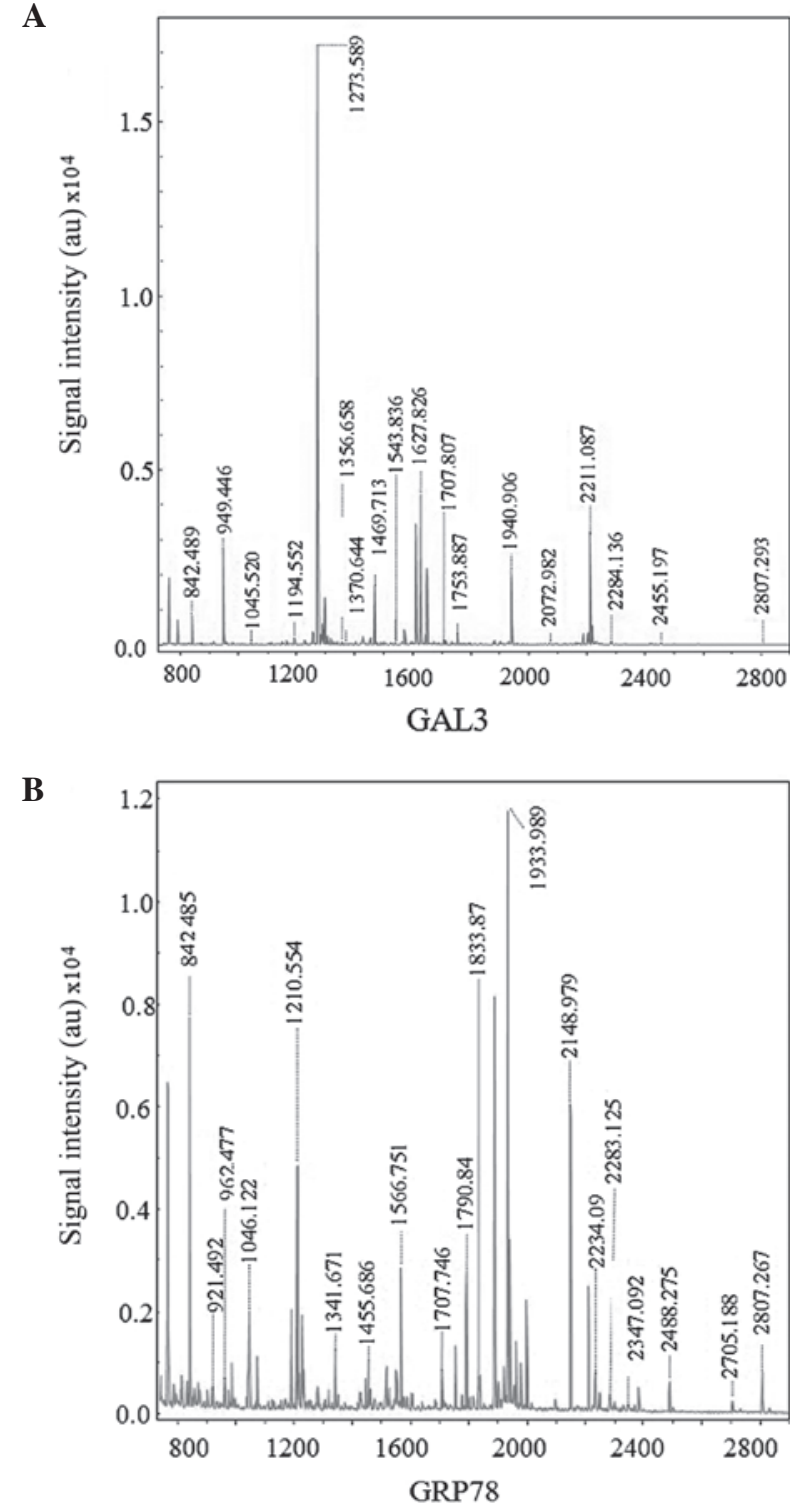

Figure 2. (A) MS analysis of GAL3. Protein score $=108$, matched peptides $=14$ and coverage $=87 \%$. (B) MS analysis of GRP78. Protein score $=143$, matched peptides $=24$ and coverage $=42 \%$. Peptide masses were determined using an Ultraflex TOF/TOF mass spectrometer. Protein scores $>82$ were considered to indicate a statistically significant difference $(\mathrm{P}<0.05)$. GAL3, galectin-3; GRP78, 78-kDa glucose-regulated protein precursor. MS, mass spectrometry; TOF, time-of-flight.

(rabbit anti-GRP78 polyclonal antibody, anti-GAL3 polyclonal antibody and mouse anti-beta actin monoclonal antibody) overnight at $4^{\circ} \mathrm{C}$. All primary antibodies were purchased from Santa Cruz Biotechnology, Inc. (Santa Cruz, CA, USA). Subsequently, membranes were washed, incubated with secondary antibodies for $1 \mathrm{~h}$ and determined using Pierce ECL western blotting kit (Thermo Fisher Scientific). The bands were analyzed using Gel-Pro Analyzer 4.0 (Media Cybernetics, Bethesda, MD, USA).

Statistical analysis. Data are presented as mean \pm SD and analyzed by the Student's t-test using SPSS version 19.0 software (SPSS, Inc., Chicago, IL, USA). P $<0.05$ was considered to indicate a statistically significant difference. 
Table I. Identification of differentially expressed proteins in Hca-F cells upon LNH treatment.

\begin{tabular}{lllrrr}
\hline No. & Protein description & NCBI GI & $\begin{array}{c}\text { Protein } \\
\text { score }\end{array}$ & $\begin{array}{c}\text { Matched } \\
\text { peptides }\end{array}$ & $\begin{array}{c}\text { Cover } \\
\text { age }(\%)\end{array}$ \\
\hline 1 & TPI & 1864018 & 165 & 15 & 66 \\
change $^{\mathrm{a}}$
\end{tabular}

${ }^{a}$ The protein spot fold change is a ratio of LNH incubation to control. LNH, lymph node homogenate. NCBI, National Center for Biotechnology Information; GI, gene identifier. TPI, triosephosphate isomerase; HP1BP3, heterochromatin protein 1 binding protein 3; GRP78, 78-kDa glucose-regulated protein precursor; DLD, dihydrolipoyl dehydrogenase; CCDC157, coiled-coil domain-containing protein 157; GAL3, galectin-3.

A

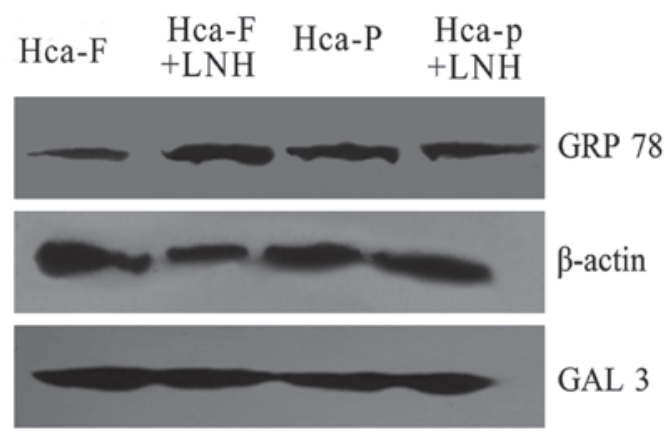

B

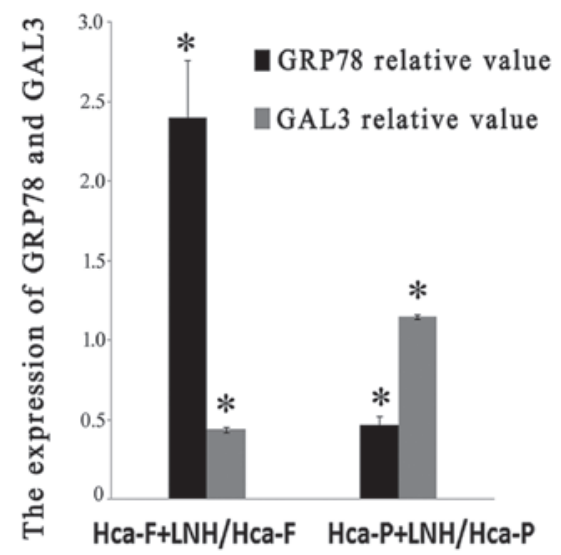

Figure 3. (A) Western blot anlaysis results of GRP78 and GAL3 expression in Hca-F cells incubated with or without LNH for $24 \mathrm{~h}$. $\beta$-actin was included as an internal protein loading control. (B) Expression difference of GRP78 and GAL3 in Hca-F and Hca-P cells upon incubation with LNH for 24 h. GRP78 was increased 2.4-fold $(\mathrm{P}<0.05)$ in Hca-F cells and decreased almost by half $(\mathrm{P}<0.05)$ in Hca-P cells. GAL3 decreased to half in Hca-F cells and increased slightly in Hca-P cells. "P<0.05. LNH, lymph node homogenates; GRP78, 78-kDa glucose-regulated protein precursor; GAL3, galectin-3.

\section{Results}

LNHs change the proteomic profiling in Hca-F cells. The extracts of Hca-F cells incubated with and without LNH were examined using 2-DE. Expression levels of 49 proteins were upregulated and 74 proteins were downregulated in Hca-F cells following cell incubation in LNH for $24 \mathrm{~h}$ (Fig. 1). Once four upregulated and four downregulated dots were detected by MALDI TOF/TOF MS (Fig. 2A and B), seven proteins were identified (Table I). Among those proteins was GRP78, an essential protein for pluripotent cell survival and embryonic cell growth. It serves as a central regulator of endoplasmic reticulum (ER) homeostasis due to its multiple functional roles in protein folding and ER calcium binding, and its control of transmembrane ER stress sensor activation $(10,11)$. GRP78 upregulation has been suggested to correlate to metastases $(12,13)$. GAL3, one of the $\beta$-galactoside-binding proteins, has been associated with cell proliferation, recognition, adhesion, differentiation, immunomodulation, apoptosis and angiogenesis. Subsequently, reduced GAL3 expression has been associated with lymph node metastasis in gastric cancer (14).

LNH differentially influences GRP78 and GAL3 expression in Hca-F and Hca-P cells on western blotting. The expression levels of GRP78 and GAL3 varied in Hca-F and Hca-P cells after culturing with LNH for $24 \mathrm{~h}$ (Fig. 3A and B). GRP78 was increased 2.4-fold $(\mathrm{P}<0.05)$ in Hca-F cells but decreased almost to half $(\mathrm{P}<0.05)$ in Hca-P cells (Fig. 3B). GAL3 decreased by half in Hca-F cells and only slightly increased in Hca-P cells (Fig. 3B). These lines of evidence suggest that following incubation with LNH the high metastatic potential cell line was induced to regulate protein expression in favor of metastases, but the low metastatic potential cell line was induced in an opposite trend against metastasis. This molecular evidence may also explain their known opposite phenotytpic metastatic characteristics in animal models.

\section{Discussion}

Organ microenvironments influence the biological behaviors of tumor cells, including cancer cell survival, proliferation, angiogenesis, invasion and metastases $(15,16)$. These studies provide important supplementary evidence for the hypothesis of metastatic niche, the modern version of 'seed and soil' 
hypothesis (17), which suggests that the intrinsic properties of the metastatic cells and the host microenvironment are important determinants in metastatic spread (7). Our results reveal that the 'non-cellular' components in LNs may regulate the cells with high metastatic potential to engage in appropriate changes for metastases. This phenomenon suggests that HCC cells with high metastatic potential demonstrate dynamic and variable superiorities compared to their low metastastic potential counterpart when they meet plausible metastatic microenvironments.

In our new model, we have used whole non-cellular components of LNs to simulate a relatively non-cellular metastatic LN microenvironment. These components, with high LNM potential, regulate the HCC cell line to express appropriate proteins necessary for LNM. Profiling/fractionation of LNH may serve to standardize our experimental conditions. We propose that within the experimental limitations of the current study, GRP78 and GAL3 may be potential biomarkers for LNM diagnosis in HCC.

\section{References}

1. Parkin DM: Global cancer statistics in the year 2000. Lancet Oncol 2: 533-543, 2001.

2. Melnikova VO and Bar-Eli M: Inflammation and melanoma metastasis. Pigm Cell Melanoma Res 22: 257-267, 2009.

3. Chu H, Zhou H, Liu Y, Hu Y and Zhang J: Functional expression of CXC chemokine recepter- 4 mediates the secretion of matrix metalloproteinases from mouse hepatocarcinoma cell lines with different lymphatic metastasis ability. Int J Biochem Cell Biol 39: 197-205, 2007

4. Zhou H, Jia L, Wang S, Wang H, Chu H, Hu Y, Cao J and Zhang J: Divergent expression and roles for caveolin-1 in mouse hepatocarcinoma cell lines with varying invasive ability. Biochem Biophys Res Commun 345: 486-494, 2006.

5. Song B, Tang JW, Wang B, Cui XN, Hou L, Sun L, Mao LM, et al: Identify lymphatic metastasis-associated genes in mouse hepatocarcinoma cell lines using gene chip. World J Gastroenterol 11: 1463-1472, 2005.
6. Langley RR and Fidler IJ: The seed and soil hypothesis revisited - the role of tumor-stroma interactions in metastasis to different organs. Int J Cancer 128: 2527-2535, 2011.

7. Bagley RG: The Tumor Microenvironment. Springer Science + Business Media, New York, 2010.

8. Jia L, Wang S, Zhou H, Cao J, Hu Y and Zhang J: Caveolin-1 upregulates CD147 glycosylation and the invasive capability of murine hepatocarcinoma cell lines. Int J Biochem Cell Biol 38: 1584-1593, 2006

9. Yang JD, Nakamura I and Roberts LR: The tumor microenvironment in hepatocellular carcinoma: current status and therapeutic targets. Semin Cancer Biol 21: 35-43, 2011.

10. Jolly C and Morimoto RI: Role of the heat shock response and molecular chaperones in oncogenesis and cell death. J Natl Cancer Inst 92: 1564-1572, 2000.

11. Luo S, Mao C, Lee B and Lee AS: GRP78/BiP is required for cell proliferation and protecting the inner cell mass from apoptosis during early mouse embryonic development. Mol Cell Biol 26: 5688-5697, 2006.

12. Luk JM, Lam CT, Siu AFM, Lam BY, Ng IO, Hu M, Che CM and Fan ST: Proteomic profiling of hepatocellular carcinoma in Chinese cohort reveals heat-shock proteins (Hsp27, Hsp70, GRP78) up-regulation and their associated prognostic values. Proteomics 6: 1049-1057, 2006.

13. Dong D, Stapleton C, Luo B, et al: A critical role for GRP78/BiP in the tumor microenvironment for neovascularization during tumor growth and metastasis. Cancer Res 71: 2848-2857, 2011.

14. Okada K, Shimura T, Suehiro T, Mochiki E and Kuwano H: Reduced galectin-3 expression is an indicator of unfavorable prognosis in gastric cancer. Anticancer Res 26: 1369-1376, 2006.

15. Fidler IJ, Kim SJ and Langley RR: The role of the organ microenvironment in the biology and therapy of cancer metastasis. J Cell Biochem 101: 927-936, 2007.

16. Fidler IJ: The organ microenvironment and cancer metastasis. Differentiation 70: 498-505, 2002.

17. Paget $S$ : The distribution of secondary growths in cancer of the breast 1889. Cancer Metastasis Rev 8: 98-101, 1989. 